\title{
Monitor Unit
}

National Cancer Institute

\section{Source}

National Cancer Institute. Monitor Unit. NCI Thesaurus. Code C112818.

A unit of measure of ionization in a linear accelerator treatment beam within the treatment head. 